\title{
The Effect of Atmospheric Plasma on the Hydrophobicity of Powdered Whey Protein Isolate
}

\author{
Heidi Lightfoot
}

Clinical Research Physician, Basingstoke and North Hampshire Hospital, England Email: heidi.lightfoot@gmail.com 


\section{Abstract:}

Whey protein is considered the most nutritionally available protein powder source. This is largely due to a variety of structural properties, one of which is hydrophobicity. Hydrophobicity is the tendency of a molecule to repel water and it plays an important role in protein bioavailability. Increasing hydrophobicity of whey protein may increase nutritional availability and thus, have various applications in the food industry. As such, techniques used to modify hydrophobicity warrant further investigation. A technique that is currently of interest is plasma-surface modification (PSM), a process that has been shown to alter the surface properties of inorganic and organic materials. In this study we explore the effect of PSM on the hydrophobicity of dry protein powder. We use 8anilinonaphtalene-1-sulfonic acid (ANS) Probe Spectrofluorometry to measure the hydrophobicity of a pristine sample of whey protein isolate and samples exposed to various atmospheric plasma conditions and demonstrate an increase in hydrophobicity with application of PSM. These findings provide further confirmation of the utility of PSM using atmospheric plasma in the food and nutrition industries.

\section{Figures:}
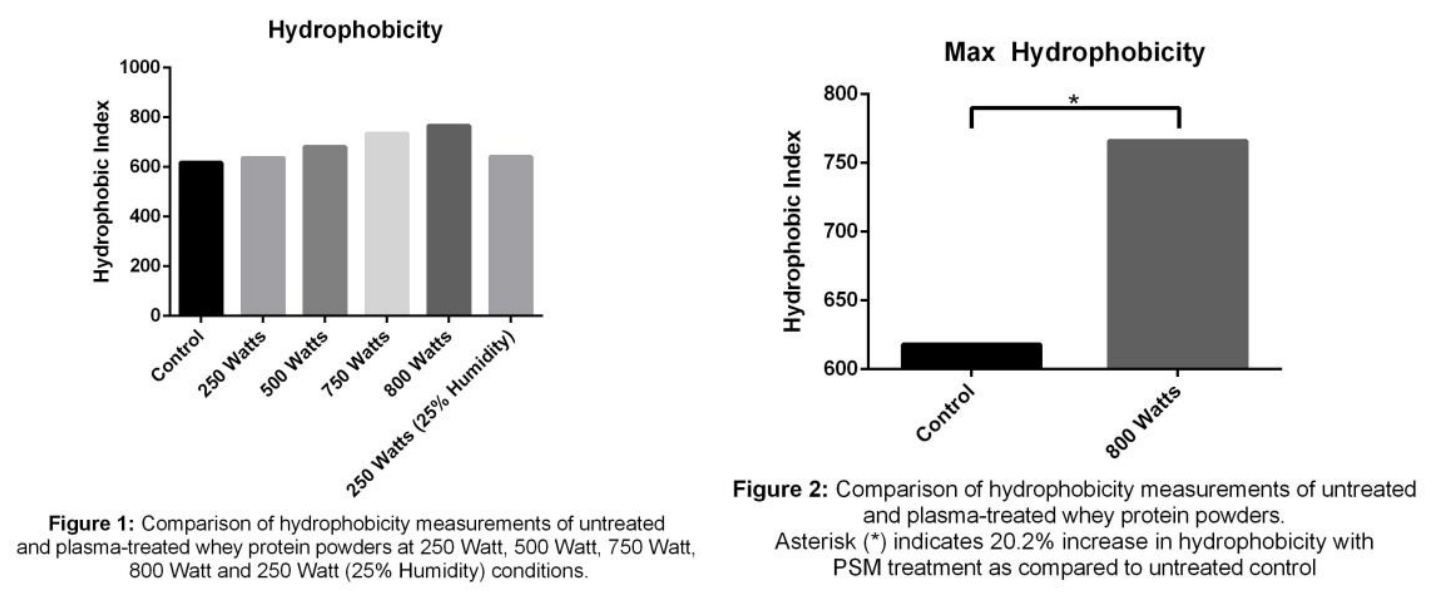


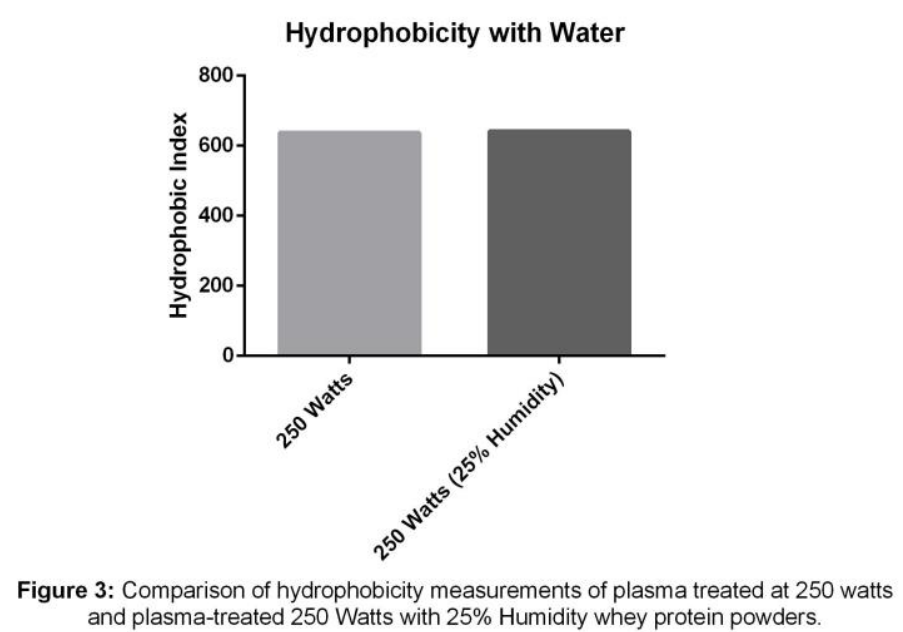

\section{Introduction:}

Whey protein is a by-product of cheese manufacturing - it is the remnant that remains after milk clotting and casein removal ${ }^{1}$. Whey protein retains approximately $55 \%$ of the milk's nutrients and makes up $20 \%$ of the total protein content of milk. ${ }^{2,3,4}$ Whey is composed of five major proteins; b-lactoglobulin, a-lactoglobulin, glycomacropeptide, proteose peptone 3 , immunoglobulins and serum albumins. ${ }^{4}$ It contains proteins, lactose, vitamins, minerals and traces of fat. These diverse constituents mean it is sold as a nutritional supplement.

Whey protein concentrate (WPC) has several additional characteristics that result in it having a number of nutritional advantages over other products. Whey is soluble, creates viscosity, forms gels, emulsifies, facilitates whipping, foaming and aeration, and it enhances color, flavor and texture. ${ }^{5}$ Measurement and quantification of protein hydrophobicity is essential to understand protein functionality, particularly with regard to food proteins. The hydrophobic, electrostatic and steric parameters of the protein structure all drive the functionality of protein molecules and protein surface hydrophobicity has been shown to be related to emulsion capacity and stability. ${ }^{6}$ Whey protein hydrophobicity has also been shown to play a pivotal role in its functionality and product applicability. ${ }^{7}$ Furthermore, increased protein hydrophobicity results in increased fat binding capacity. ${ }^{8}$ Therefore, it is safe to say that changes that affect or enhance protein hydrophobicity have the potential to improve the flavor properties of foods.

Plasma surface modification (PSM) is a common technique used to alter surface properties of inorganic materials in chemical engineering with various applications in the medical field. Applying this process to organic materials offers the potential to optimize the surface characteristics described above and improve the function of the target material in an economical and reliable fashion. Previous findings have demonstrated the 
utility of PSM in increasing the surface area of whey protein powder using atmospheric plasma. The use of PSM to alter the hydrophobicity of powdered whey isolate has yet to be studied but could prove to be an important tool to improve the nutritional content of food.

\section{Objective:}

This study compares the hydrophobicity of untreated and PSM-treated powdered whey isolate.

\section{Results \& Discussion:}

A commercially available dry powder whey protein isolate was used as the source material for the study. This also served as the control for the experiment. Portions of this isolate batch were separated and exposed to atmospheric plasma treatment at several wattage settings. Proprietary PSM technology (Ingredient Optimization ${ }^{\mathrm{TM}}$, Plasma Nutrition, USA) was used to mediate the exposure of organic dry powder materials to plasma to induce several changes, including surface-level alterations.

Our hypothesis was tested by investigating changes in hydrophobic indices following PSM using ANS Probe Spectrofluorometry analysis. We found that untreated whey protein powder had a lower hydrophobic index than any of the PSM treated samples (Figures 1,2). Specifically, the hydrophobic index of the control powder was 618, while those of the treated powders were 637, 641, 681, 735, and 766 (depending on the wattage setting). Exposure of the dry powder whey protein isolate to atmospheric plasma yielded an overall increase in hydrophobicity by up to $20.2 \%$ (Figure 2). Interestingly, hydrophobic indices were different for samples with identical plasma conditions but altered reaction humidity (Figures 1, 3). In other words, powder batches that were treated under the same wattage but different humidity conditions resulted in different hydrophobicity indices (Figures 1,3). This may suggest that plasma, under favorable conditions, could be an economical method for protein hydrolysis. However, further investigations into this are warranted to fully establish the mechanics behind this finding.

As previously mentioned, understanding protein hydrophobicity helps explain its functionality. Furthermore, increasing food protein hydrophobicity has been shown to enhance its binding capacity and as such can be associated with improvement of food and nutrition products. ${ }^{12}$ We demonstrate that PSM is an effective method for enhancing whey protein hydrophobicity. Our findings suggest its application could be of use in the optimization of whey protein as a food and nutrition substitute, which could be of great commercial and economical use in the food and nutrition industries.

\section{Conclusions:}


In this study, changes in hydrophobicity of untreated and PSM treated dry powdered whey protein isolate samples from the same commercially available powder batch were compared. An increase in the hydrophobicity of the samples that had undergone PSM was observed. This is in line with our expectations, as the results are consistent with previously observed effects of PSM on other substrates such as metals and glass.

In conclusion, PSM increases the hydrophobicity of dry powdered whey protein isolate, and thus it can be inferred that organic materials such as whey protein are candidates for surface engineering via atmospheric plasma. Our findings validate the use of PSM as a means to enhance hydrophobicity and thus potentially improve absorption of substances like dry protein powder that is widely used in food production.

\section{Methods:}

Commercially available whey protein powder was obtained and a sample to be used as the control was separated prior to processing. Using proprietary PSM technology (Ingredient Optimization TM, Plasma Nutrition, USA), samples were fed through a reaction chamber and exposed to $250 \mathrm{~W}, 500 \mathrm{~W}, 750 \mathrm{~W}$ or $800 \mathrm{~W}$ of atmospheric plasma. During this process, the whey powder was aerated to maximize the surface area of the protein exposed to the plasma.

Following the protocol previously described in the literature by XX et. al (Measurement of Protein Hydrophobicity text chapter), duplicate $2 \mathrm{~mL}$ serial dilution aliquots of protein solutions ranging from $0.005 \%$ to $0.03 \%(\mathrm{w} / \mathrm{v})$ were prepared in $0.01 \mathrm{M}$ phosphate buffer $(\mathrm{pH} 5.5$ to 7.4), containing 0 to $0.6 \mathrm{M} \mathrm{NaCl}$. A total of $10 \mathrm{uL}$ of $8.0 \mathrm{mM} 1$-anilinonaphthalene-8-sulfonic acid in $0.1 \mathrm{M}$ phosphate buffer $(\mathrm{pH} 7.0)$ was added to one serial dilution aliquot set, and 10uL of the solvent used to prepare the probe solution was added to the other serial dilution aliquot set as a control (blank).

Spectrofluorometric measurements were obtained using a SPECTROstar Omega (BMG LabTech, Ortenberg, Germany). The spectrofluorometer was standardized by adjusting the reading for equivalent concentrations of ANS in methanol to an arbitrary value (i.e. at $70 \%$ of the full scale). Relative fluorescence intensity (RFI) of each sample and control was obtained at excitation $(\lambda \mathrm{em}=390 \mathrm{~nm})$, and emission $(\lambda \mathrm{em}=470 \mathrm{~nm})$ wavelengths and the RFI for each sample concentration was computed after subtracting the corresponding blank values. RFI versus protein concentration values were plotted and linear regression analyses were used to find the slopes (SO) that represented the hydrophobic indices. Data was analyzed using Prism 6 (GraphPad Software). A paired nonparametric one-way ANOVA was performed yielding results with a $\mathrm{P}<0.05$ $(\mathrm{P}=0.0052)$. 


\section{Limitations:}

This study investigated the application of PSM on only one source of dry powdered whey protein isolate. While the whey protein isolate source material used in this investigation is commonly found in commercial use, there may be variations between the material used and other market-available whey protein isolate powders. Further studies are needed to examine the effect of PSM on a range of dry powder whey protein isolate sources.

\section{Funding Statement:}

This work was financially supported by Plasma Nutrition.

\section{Ethics Statement:}

All experiments were performed in vitro and did not involve the use of any living subject. No fraudulence is committed in performing these experiments or during processing of the data. We understand that in the case of fraudulence, the study can be retracted by Matters.

\section{Sources:}

1. Brandelli, A., Daroit, D.J. and Corrêa, A.P.F. (2015). Whey as a source of peptides with remarkable biological activities. Food Research International, 73, pp.149-161. DOI: 10.1016/j.foodres.2015.01.016

2. Siso, M.G. (1996). The biotechnological utilization of cheese whey: a review. Bioresource technology, 57(1), pp.1-11. DOI: 10.1016/09608524(96)00036-3

3. Smithers, G.W. (2008). Whey and whey proteins-from 'gutter-togold'. International Dairy Journal, 18(7), pp.695-704. DOI: 10.1016/j.idairyj.2008.03.008

4. Krissansen, G.W. (2007). Emerging health properties of whey proteins and their clinical implications. Journal of the American College of Nutrition, 26(6), pp.713S723S. DOI: 10.1080/07315724.2007.10719652

5. U.S. Dairy Export Council. (1999). Product specifications. Arlington, VA, USA' U.S. Dairy Export Council

6. Nakai, S. (1983). Structure - function relationships of food proteins with an emphasis on the importance of protein hydrophobicity. Journal of Agricultural and Food Chemistry, 31, 676 - 683. DOI: 10.1021/jf00118a001

7. Mangino, M.E., Huffman, L.M. and Regester, G.O. (1988). Changes in the hydrophobicity and functionality of whey during the processing of whey protein concentrates. Journal of Food Science, 53(6), pp.1684-1686. DOI:

10.1111/j.1365-2621.1988.tb07815.x 
8. Voutsinas, L.P. and Nakai, S. (1983). A simple turbidimetric method for determining the fat binding capacity of proteins. Journal of Agricultural and Food Chemistry, 31(1), pp.58-63. DOI: 10.1021/jf00115a015

9. Chu, P.K., Chen, J.Y., Wang, L.P. and Huang, N. (2002). Plasma-surface modification of biomaterials. Materials Science and Engineering: $R$ : Reports, 36(5), pp.143-206. DOI: 10.1016/S0927-796X(02)00004-9

10. Chao, C.C., Ma, Y.S. and Stadtman, E.R. (1997). Modification of protein surface hydrophobicity and methionine oxidation by oxidative systems. Proceedings of the National Academy of Sciences, 94(7), pp.2969-2974.

11. del Castillo-Santaella, T., Sanmartín, E., Cabrerizo-Vílchez, M.A., Arboleya, J.C. and Maldonado-Valderrama, J. (2014). Improved digestibility of $\beta$-lactoglobulin by pulsed light processing: a dilatational and shear study. Soft matter, 10(48), pp.9702-9714. DOI: 10.1039/C4SM01667J

12. Liu, X., Powers, J.R., Swanson, B.G., Hill, H.H. and Clark, S. (2005). Modification of whey protein concentrate hydrophobicity by high hydrostatic pressure. Innovative Food Science \& Emerging Technologies, 6(3), pp.310-317. DOI: 10.1016/j.ifset.2005.03.006 